\title{
Survival exponents for fractional Brownian motion with multivariate time
}

\section{George Molchan}

Institute of Earthquake Prediction Theory and Mathematical Geophysics, Russian Academy of Science, 84/32 Profsoyuznaya st., 117997, Moscow, Russian Federation.

E-mail address: molchan@mitp.ru

\begin{abstract}
Fractional Brownian motion of index $0<H<1, H$-FBM, with $d$ dimensional time is considered in a spherical domain that contains 0 at its boundary. The main result: the log-asymptotics of the probability that H-FBM does not exceed a fixed positive level is $(H-d+o(1)) \log T$, where $T>>1$ is the radius of the domain.
\end{abstract}

\section{Introduction}

Fractional Brownian motion of index $H \in(0,1)$, H-FBM, with multivariate time $t \in R^{d}$ is a centered Gaussian random process $w_{H}(t)$ with correlation function

$$
E w_{H}(t) w_{H}(s)=0.5\left(|t|^{2 H}+|s|^{2 H}-|t-s|^{2 H}\right) .
$$

H-FBM is H-self-similar (H-ss), isotropic, and has stationary increments (si), i.e.,

$$
\left\{w_{H}\left(\lambda U t+t_{0}\right)-w_{H}\left(t_{0}\right)\right\} \doteq\left\{\lambda^{H} w_{H}(t)\right\}
$$

holds in the sense of the equality of finite-dimensional distributions for any fixed $t_{0}, \lambda>0$, and orthogonal mapping $U: R^{d} \rightarrow R^{d}$.

The one-sided exit problem for a random process $\xi(t)$ and its characteristics, the so-called survival exponents:

$$
\theta_{\xi}=\lim _{T \rightarrow \infty}-\log P\left(\xi(t)<1, t \in \Delta_{T}\right) / \psi(T)
$$

are the subject of intensive analysis in applications. Here $\Delta_{T}$ is an increasing sequence of domains of size $T$, and $\psi(\cdot)$ is a suitable slowly varying function, typically, $\psi(T)=\log T$ for ss-processes. The greatest progress in this area has been achieved for processes with one-dimensional time. (See surveys by Bray et al., 2013 of the

Received by the editors September 27, 2016; accepted December 2, 2016.

2010 Mathematics Subject Classification. 60G22.

Key words and phrases. Fractional Brownian motion; One-sided exit problem; Survival exponent.

Research supported by Russian Science Foundation, grant 17-11-01052. 
physics literature and by Aurzada and Simon, 2015 of the relevant mathematical publications).

H-FBM was one of the first non-trivial examples of non-Markovian processes for which the survival exponents have been found exactly (Molchan, 1999). Namely, for $\psi(T)=\log T$, the survival exponents for H-FBM are

$$
\theta_{w_{H}}=1-H, \quad \Delta_{T}=(0, T) \quad \text { and } \quad \theta_{w_{H}}=d, \quad \Delta_{T}=(-T, T)^{d} .
$$

Recently, Aurzada et al. (2016) considerably refined the asymptotics of probability

$$
p_{T}=P\left(w_{H}(t)<1, t \in \Delta_{T}\right), \quad \Delta_{T}=(0, T)
$$

and showed that the exponent $\theta=1-H$ is universal for a broad class of H-ss processes with stationary increments. The ideas of this work have proved useful in the analysis of the conjecture that $\theta_{w_{H}}=d-k H$ for $w_{H}(t)$ in $\Delta_{T}=[0, T]^{k} \times$ $[-T, T]^{d-k}$ (Molchan, 2012).

The case $k=0$ corresponds to the right part of (1.2). The case $k=1$ is supported by the result which we discuss below: $\theta_{w_{H}}=d-H$ for fractional Brownian motion in $\Delta_{T}=T \Delta_{1}$, where $\Delta_{1}$ is a unit ball that contains 0 at its boundary.

The main idea of the paper by Aurzada et al. (2016) is to show that for a broad class of si-processes, $\xi(t), \xi(0)=0$, with discrete time

$$
\left|\Delta_{T}\right| P\left(\xi(t)<1, t \in \Delta_{T} \cap \mathbb{Z}^{1}\right) \approx E \max \left(\xi(t), t \in \Delta_{T} \cap \mathbb{Z}^{1}\right),
$$

where $\Delta_{T}=[0, T],\left|\Delta_{T}\right|=T$, and $\approx$ means up to a multiplicative term in $T^{o(1)}$. For $\mathrm{H}$-ss processes with continuous time, the right-hand part of (1.4) is proportional to $T^{H}$, and therefore the exponent for (1.3) is $1-H$. However, the result by Aurzada et al. (2016) essentially uses the 1-D nature of time. Considering $\left|\Delta_{T}\right|$ as the volume of $\Delta_{T}$, relation (1.4) is found to be in formal agreement with the conjecture for $k=1$, but not for $k>1$; in addition, (1.4) is very crude for $k=0$ (see (1.3)). This means that the analysis of the cases $d>1, k>1$ needs more ideas.

\section{The lower bound}

Proposition 2.1. Let $\xi(t), \xi(0)=0, t \in R^{d}$ be a centered isotropic random process with stationary increments. Then

$$
P\left(\xi(t)<-1, t \in \Delta_{T},|t|>1\right) \leq c T^{-d} E \max \left(\xi(t), t \in \Delta_{T}\right),
$$

where $\Delta_{T}=T \Delta_{1}$ is a ball of radius $T$ that contains 0 at its boundary.

Consequence 2.2. If $\xi(t)$ is fractional Brownian motion of index $H \in(0,1)$ in $\Delta_{T}$, then the survival exponent has the lower bound $\theta_{w_{H}}^{-} \geq d-H$.

Remark 2.3. Proposition 2.1 holds for $\Delta_{T}=[0, T] \times[-T, T]^{d-1}$ as well.

Proof: Let $U_{T}=\left\{x_{k, \alpha}, \alpha=1,2, \ldots, n_{k} ; k=1,2, \ldots\right\}$ be a subset of ball $B_{T}$ of radius $T$ in $R^{d} ; U_{T}$ consists of $N_{T}$ points such that

$$
\left|x_{k, \alpha}\right|=r_{k}, \quad\left|x_{k, \alpha}-x_{m, \beta}\right|>1, \quad N_{T}>C T^{d}, \quad 1<r_{k}<r_{k+1} \leq T .
$$

Consider the following increasing sequence of subsets of $U_{T}$ :

$$
U_{k+1, \alpha}=U_{k} \cup \bigcup_{\beta=1}^{\alpha} x_{k+1, \beta}, \quad U_{k}=\left\{x_{i, \gamma}:\left|x_{i, \gamma}\right| \leq r_{k}\right\}
$$


Fix $\Delta_{T}=\{t:|t+T e| \leq T\}$, where $e=(0, \ldots, 0,1)$. Let $O_{k, \alpha}$ be an orthogonal mapping transforming $x_{k, \alpha}$ in $\tilde{x}_{k, \alpha}=r_{k} e$. Setting $\tilde{U}_{k, \alpha}=O_{k, \alpha} U_{k, \alpha}$, one has

$$
\left(\tilde{U}_{k, \alpha}-\tilde{x}_{k, \alpha}\right) \backslash\{0\} \subset \Delta_{T} \backslash B_{1}, \quad(k, \alpha) \neq(1,1) .
$$

Therefore, using the notation $M(A)=\sup (\xi(t), t \in A)$, we get

$$
p_{T}(-1):=P\left(\xi(t)<-1, t \in \Delta_{T} \backslash B_{1}\right) \leq P\left(M\left(\left(\tilde{U}_{k, \alpha}-\tilde{x}_{k, \alpha}\right) \backslash\{0\}\right)<-1\right) .
$$

By the si-property of $\xi(t)$, we can continue

$$
=P\left(M\left(\tilde{U}_{k, \alpha} \backslash \tilde{x}_{k, \alpha}\right)-\xi\left(\tilde{x}_{k, \alpha}\right)<-1\right)=P\left(M\left(U_{k, \alpha-1}\right)+1<\xi\left(x_{k, \alpha}\right)\right) .
$$

The last equality holds because $\xi(t)$ is rotation invariant.

The event $\left\{M\left(U_{k, \alpha-1}\right)+1<\xi\left(x_{k, \alpha}\right)\right\}$ is measurable relative to the sequence

$$
\xi\left(x_{1,1}\right), \ldots, \xi\left(x_{1, n_{1}}\right) ; \ldots ; \xi\left(x_{k, 1}\right), \ldots, \xi\left(x_{k, n_{k}}\right) ; \ldots
$$

This event take place when $\xi\left(x_{k, \alpha}\right)$ is realized as a record in the sequence (2.5) which exceeds the previous one by at least 1 . Let $\nu_{T}$ be the number of such records in $(2.5)$. Then, by $(2.3,2.4)$,

$$
\left(N_{T}-1\right) p_{T}(-1) \leq \sum_{k, \alpha} P\left(M\left(U_{k, \alpha}\right)+1<\xi\left(x_{k, \alpha+1}\right)\right)=E \nu_{T} \leq E\left(M\left(U_{T}\right)-\xi\left(x_{1,1}\right)\right),
$$

where $U_{1,1}=\left\{x_{1,1}\right\}, \quad U_{k, n_{k}}=U_{k+1}, \quad x_{k, n_{k}+1}=x_{k+1,1}, \quad(k, \alpha) \neq(1,1)$.

Finally, by (2.1),

$$
p_{T}(-1) \leq E\left(M\left(U_{T}\right)\right) /\left(N_{T}-1\right)<c T^{-d} E\left(\sup \xi(t), t \in \Delta_{T}\right) .
$$

Suppose that $\xi(t)$ is fractional Brownian motion of index $H \in(0,1)$ in $\Delta_{T}$. By the standard procedure, we can compare $p_{T}(-1)$ with

$$
p_{T}(1)=P\left(w_{H}(t)<1, t \in\left(\Delta_{T} \backslash B_{1}\right)\right) .
$$

For this purpose we can find a continuous function $\varphi_{T}(t)$ such that

$$
\varphi_{T}(t)=1,|t|>1,\left\|\varphi_{T}\right\|_{H, T}<\text { const },
$$

where $\|\cdot\|_{H, T}$ is the norm of the Hilbert space $H_{H}\left(\Delta_{T}\right)$ with the reproducing kernel $E w_{H}(t) w_{H}(s),(t, s) \in \Delta_{T} \times \Delta_{T}$ (see for this fact Molchan, 1999 or Appendix). Then

$$
p_{T}(-1)=P\left(w_{H}(t)+2 \varphi_{T}(t)<1, t \in\left(\Delta_{T} \backslash B_{1}\right)\right) .
$$

According to Aurzada and Dereich (2013),

$$
\left|\sqrt{\ln 1 / p_{T}(1)}-\sqrt{\ln 1 / p_{T}(-1)}\right| \leq\left\|2 \varphi_{T}\right\|_{H, T} / \sqrt{2} .
$$

From the self-similarity of H-FBM and (2.6) one has

$$
p_{T}(-1) \leq c T^{-(d-H)} E M_{w_{H}}\left(\Delta_{1}\right) .
$$

Combining (2.8-2.10), one has

$$
\left[\ln 1 / P\left(w_{H}(t)<1, t \in \Delta_{T}\right)\right]^{1 / 2} / \sqrt{\ln T} \geq \sqrt{d-H}+O(1 / \sqrt{\ln T}),
$$

i.e., $\theta_{w_{H}} \geq d-H$. 


\section{The upper bound}

Below we use notation $M(A)=\sup \left(w_{H}(t), t \in A\right)$ and $|A|=\#\{t: t \in A\}$.

Proposition 3.1. Let $w_{H}(t)$ be H-FBM in $\Delta_{T}=T \Delta_{1} \subset R^{d}$ where $\Delta_{1}$ is a bounded domain and $0 \in \Delta_{1}$. Consider a finite 1-net of $\Delta_{T}$, i.e. a subset $U_{T}=\left\{x_{k}, k=\right.$ $\left.1, \ldots, N_{T}\right\} \subset \Delta_{T},\{0\} \notin U_{T}$ such that

$$
c<N_{T} / T^{d}<C \quad \text { and } \quad \Delta_{T} \subset \bigcup_{r=1}^{N_{T}} B_{1}\left(x_{r}\right),
$$

where $B_{1}(x)$ is a unit ball centered at $x$. Then there is a $0<q<1$ such that for all $T>T_{0}$

$$
P\left(M\left(\Delta_{T}\right)<c_{H} \sqrt{4 d \ln T}\right) \geq q P\left(M\left(U_{T}\right)<0\right) .
$$

In addition,

$$
E M\left(U_{T}\right)=E M\left(\Delta_{T}\right)(1+o(1))=T^{H} E M\left(\Delta_{1}\right)(1+o(1)), T \rightarrow \infty .
$$

Proof: One has

$$
P\left(M\left(U_{T}\right)<0\right) \leq P\left(M\left(U_{T}\right)<0, A_{T}\right)+P\left(A_{T}^{c}\right),
$$

where

$$
A_{T}=\left\{\max _{k} \max _{t}\left(w_{H}(t)-w_{H}\left(x_{k}\right), t \in B_{1}\left(x_{k}\right)\right)<b_{T}\right\} .
$$

We can continue the previous inequality

$$
\begin{aligned}
& \leq P\left(M\left(\Delta_{T}\right)<b_{T}\right)+\sum_{k} P\left(\max \left(w_{H}(t)-w_{H}\left(x_{k}\right), t \in B_{1}\left(x_{k}\right)\right)>b_{T}\right) \\
& \leq P\left(M\left(\Delta_{T}\right)<b_{T}\right)+N_{T} P\left(M\left(B_{1}\right)>b_{T}\right):=p_{1, T}+p_{2, T} .
\end{aligned}
$$

Applying the Fernique (1975) inequality to $w_{H}(t)$, we have

$$
P\left(M\left(B_{1}\right)>r_{T} c_{H}\right) \leq c_{d} \int_{r_{T}}^{\infty} e^{-u^{2} / 2} d u, \quad r_{T}>(1+4 d)^{1 / 2}
$$

Hence, setting $b_{T}=\sqrt{2(2 d+\varepsilon) \ln T} c_{H}, \varepsilon>0$, one has

$$
p_{2, T}<C T^{d} \cdot T^{-2 d-\varepsilon} / \sqrt{\ln T}=C T^{-d-\varepsilon} / \sqrt{\ln T} .
$$

To show that $p_{2, T}=o\left(p_{1, T}\right)$, note that $\Delta_{T} \subset B_{T D}$, where $D$ is the diameter of $\Delta_{1}$. Therefore

$$
p_{1, T}=P\left(M\left(\Delta_{T}\right)<b_{T}\right) \geq P\left(M\left(B_{T D}\right)<b_{T}\right)=P\left(M\left(B_{T^{\prime}}\right)<1\right),
$$

where $T^{\prime}=T D / b_{T}^{1 / H}$. By Molchan (1999),

$$
P\left(M\left(B_{T}\right)<1\right)>c T^{-d-\varepsilon} .
$$

Due to (3.6), (3.7), we have

$$
p_{2, T} / p_{1, T}<c(\ln T)^{-(1+d / H) / 2}=o(1) .
$$

Relations (3.3, 3.4) and (3.8) imply (3.1):

$$
P\left(M\left(U_{T}\right)<0\right) \leq(1+o(1)) p_{1, T} \leq(1+\varepsilon) P\left(M\left(\Delta_{T}\right)<b_{T}\right),
$$

where $b_{T}=\sqrt{4 d \ln T} c_{H}$. To prove relation (3.2), note that

$$
\begin{aligned}
M\left(\Delta_{T}\right) & \leq M\left(U_{T}\right)+\max _{k} \max _{t}\left(w_{H}(t)-w_{H}\left(x_{k}\right), t \in B_{1}\left(x_{k}\right)\right) \\
& :=M\left(U_{T}\right)+\delta_{T} .
\end{aligned}
$$


As above, using the event $A_{T}=\left\{\max _{k} \max _{t}\left(w_{H}(t)-w_{H}\left(x_{k}\right), t \in B_{1}\left(x_{k}\right)\right)<b_{T}\right\}$, one has

$$
E \delta_{T} \leq b_{T}+E \delta_{T} 1_{A_{T}^{c}} \leq b_{T}+N_{T} E M\left(B_{1}\right)\left[M\left(B_{1}\right)>b_{T}\right],
$$

where $b_{T}=\sqrt{4 d \ln T} c_{H}$ and $N_{T}<C T^{d}$.

Therefore, the second term in (3.9) is $o(1)$, because

$$
\left(E M\left(B_{1}\right)\left[M\left(B_{1}\right)>b_{T}\right]\right)^{2} \leq E M^{2}\left(B_{1}\right) P\left(M\left(B_{1}\right)>b_{T}\right)=O\left(T^{-2 d} / \sqrt{\ln T}\right) .
$$

Due to (3.9), the relation (3.2) follows from the inequality:

$$
\begin{aligned}
E M\left(U_{T}\right) & \geq E M\left(\Delta_{T}\right)-E \delta_{T} \geq E M\left(\Delta_{T}\right)-c \sqrt{\ln T}+o(1) \\
& =T^{H} E M\left(\Delta_{1}\right)-c \sqrt{\ln T}+o(1) .
\end{aligned}
$$

Proposition 3.2. Let $w_{H}(t), t \in \Delta_{T}$ be H-FBM, $\Delta_{T}=T \Delta_{1} \subset R^{d}$, where $\Delta_{1}$ is a unit ball and $0 \in \Delta_{1}$. Then

$$
P\left(M\left(\Delta_{T}\right)<1\right) \geq c T^{-(d-H)}(\sqrt{\ln T})^{-d / H},
$$

i.e., the survival exponent for $H-F B M$ in $\Delta_{T}$ has the upper bound $\theta_{w_{H}}^{+} \leq d-H$.

Corollary 3.3. Due to Propositions 2.1, 3.2, the survival exponent for H-FBM in $\Delta_{T}$ exists and equals $d-H$.

Proof: Proceeding as in the proof of Proposition 2.1, we consider again the subset $U_{T}$ of the ball $B_{T} \subset R^{d}: U_{T}=\left\{x_{k, \alpha}, \alpha=1,2, \ldots, n_{k} ; k=1,2, \ldots\right\},\{0\} \notin U_{T}$. In addition to the properties (2.1), we suppose that the elements of $U_{T}$ are enumerated in such a way that

$$
x_{k, \alpha+1} \in B_{2}\left(x_{k, \alpha}\right) \quad \text { and } \quad x_{k+1,1} \in B_{2}\left(x_{k, n(k)}\right) .
$$

As before,

$$
U_{k+1, \alpha}=U_{k} \cup \bigcup_{\beta=1}^{\alpha} x_{k+1, \beta}, U_{k}=\left\{x_{i, \gamma}:\left|x_{i, \gamma}\right| \leq r_{k}\right\}:=U_{k, 0} ;
$$

$\Delta_{T}=\{t:|t+T e| \leq T\}$, where $e=(0, \ldots, 0,1) ; O_{k, \alpha}$ is an orthogonal mapping transforming $x_{k, \alpha}$ in $\tilde{x}_{k, \alpha}=r_{k} e$. Setting $\tilde{U}_{k, \alpha}=O_{k, \alpha} U_{k, \alpha}$, one has

$$
\left(\tilde{U}_{k+1, \alpha}-\tilde{x}_{k+1, \alpha}\right) \backslash\{0\} \subset \Delta_{k+1} \backslash B_{1} .
$$

Due to (3.10), $\left(\tilde{U}_{k+1, \alpha}-\tilde{x}_{k+1, \alpha}\right)$ is a 2 -net in $\Delta_{k+1}$. Therefore, by (3.1), for $k>T_{0}$

$$
\begin{gathered}
\left.P\left(M\left(\Delta_{k}\right)<c_{H} \sqrt{4 d \ln k}\right)>q P\left(M\left(\tilde{U}_{k, \alpha}-\tilde{x}_{k, \alpha}\right) \backslash\{0\}\right)<0\right) \\
\left.=q P\left(M\left(\tilde{U}_{k, \alpha-1}\right)-w_{H}\left(\tilde{x}_{k, \alpha}\right)\right)<0\right)=q P\left(M\left(U_{k, \alpha-1}\right)<w_{H}\left(x_{k, \alpha}\right)\right) .
\end{gathered}
$$

As a result,

$$
\sum_{k=K}^{K^{\prime}} n_{k} P\left(M\left(\Delta_{k}\right)<c_{H} \sqrt{4 d \ln k}\right) \geq q \sum_{k=K}^{K^{\prime}} \sum_{\alpha=1}^{n_{k}} P\left(M\left(U_{k, \alpha-1}\right)<w_{H}\left(x_{k, \alpha}\right)\right)
$$

where $K=[T]$ and $K^{\prime}=\left[T^{\prime}\right]$.

Similarly to the proof of Proposition 2.1, we conclude that the right-hand part of (3.11) is equal to $q E \nu\left(T, T^{\prime}\right)$, where $\nu\left(T, T^{\prime}\right)$ is the number of all records in the following sequences:

$$
M\left(U_{K}\right), w_{H}\left(x_{K+1,1}\right), \ldots, w_{H}\left(x_{K+1, n(K+1)}\right) ; \ldots ; w_{H}\left(x_{K^{\prime}, 1}\right), \ldots, w_{H}\left(x_{K^{\prime}, n\left(K^{\prime}\right)}\right) .
$$


Let $\delta\left(T, T^{\prime}\right)$ be the maximum increment between adjacent elements of the sequence $w_{H}\left(x_{K, n(K)}\right), w_{H}\left(x_{K+1,1}\right), \ldots, w_{H}\left(x_{K+1, n(K+1)}\right) ; \ldots ; w_{H}\left(x_{K^{\prime}, 1}\right), \ldots$, $w_{H}\left(x_{K^{\prime}, n\left(K^{\prime}\right)}\right)$. Then

$$
M\left(U_{K^{\prime}}\right)-M\left(U_{K}\right) \leq\left(\nu\left(T, T^{\prime}\right)+1\right) \delta\left(T, T^{\prime}\right) \leq\left(\nu\left(T, T^{\prime}\right)+1\right) b_{T}+R_{T},
$$

where

$$
R_{T}=\left(\left|U_{K^{\prime}} \backslash U_{K}\right|+1\right) \delta\left(T, T^{\prime}\right)\left[\delta\left(T, T^{\prime}\right)>b_{T}\right]
$$

Due to (3.10),

$$
E R_{T}<\left(\left|U_{K^{\prime}} \backslash U_{K}\right|+1\right)^{2} \max _{|t|<2} E w_{H}(t)\left[w_{H}(t)>b_{T}\right] .
$$

Setting $b_{T}=\sqrt{8 d \ln T} c_{H}$ and $T^{\prime}-T=\rho T$, we obtain

$$
E R_{T}<c T^{2 d} \cdot T^{-2 d}=c .
$$

By (3.12),

$$
b_{T} E \nu\left(T, T^{\prime}\right)>E M\left(U_{K^{\prime}}\right)-E M\left(U_{K}\right)-b_{T}-E R_{T},
$$

where, according to (3.2),

$$
\operatorname{EM}\left(U_{K}\right)=K^{H} \operatorname{EM}\left(\Delta_{1}\right)(1+o(1)) .
$$

As a result,

$$
b_{T} E \nu\left(T, T^{\prime}\right)>c\left(T^{H}-\sqrt{\ln T}-1\right)=c T^{H}(1+o(1)) .
$$

Keeping in mind that the right part of (3.11) is $q E \nu\left(T, T^{\prime}\right)$, we have:

$$
q E \nu\left(T^{\prime}, T\right) \leq \sum_{k=K}^{K^{\prime}} n_{k} P\left(M\left(\Delta_{k}\right)<c_{H} \sqrt{4 d \ln k}\right) .
$$

Due to the self-similarity of H-FBM,

$$
P\left(M\left(\Delta_{k}\right)<c \sqrt{\ln k}\right)=P\left(M\left(\Delta_{\tilde{k}}\right)<1\right), \quad \tilde{k}=k /(c \sqrt{\ln k})^{1 / H},
$$

and therefore the probability term decreases as a function of $k$. Hence, (3.15) implies

$$
\begin{aligned}
q E \nu\left(T^{\prime}, T\right) & \leq\left|U_{T^{\prime}} \backslash U_{T}\right| P\left(M\left(\Delta_{T^{\prime}+1}\right)<c_{H} \sqrt{4 d \ln \left(T^{\prime}+1\right)}\right) \\
& \leq C T^{d} P\left(M\left(\Delta_{\tilde{T}}\right)<1\right)
\end{aligned}
$$

where

$$
\tilde{T}=T^{\prime} /\left(c_{H} \sqrt{4 d \ln T^{\prime}}\right)^{1 / H} \quad \text { or } \quad T^{\prime}=\tilde{T}\left(c_{H} \sqrt{4 d \ln \tilde{T}}\right)^{1 / H}(1+o(1)) .
$$

Finally, by $(3.14,3.16)$,

$$
b_{T}^{-1} c T^{H}(1+o(1)) \leq E \nu\left(T^{\prime}, T\right) \leq q^{-1} C T^{d} P\left(M\left(\Delta_{\tilde{T}}\right)<1\right) .
$$

Taking into account (3.17) and the relation $T^{\prime}-T=\rho T$, we get

$$
P\left(M\left(\Delta_{\tilde{T}}\right)<1\right) \geq c \tilde{T}^{-(d-H)}(\sqrt{\ln \tilde{T}})^{-d / H} .
$$




\section{Appendix}

Example from Proposition 2.1. Consider H-FBM in domains $\Delta_{T}=T \cdot \Delta_{1}$, $0 \in \partial \Delta_{1}$; then a suitable function $\varphi_{T}(t), t \in \Delta_{T}$ can be chosen as follows:

$$
\varphi_{T}(t)=f(|t| /(T k))-f(|t|),
$$

where $f(x), x \in R^{1}$ is a finite smooth function such that $f(t)=1$ for $|x|<1 / 2$ and $f(t)=0$ for $|x|>1$. Here $k$ is the diameter of $\Delta_{1}$.

By Molchan (1999), this can be seen as follows. Due to the spectral representation of H-FBM, the Hilbert space $H_{H}\left(\Delta_{T}\right)$ with the reproducing kernel $E w_{H}(t) w_{H}(s),(t, s) \in \Delta_{T} \times \Delta_{T}$ (Lifshits, 2012), is the closure of smooth functions $\varphi(t), \varphi(0)=0$ relative to the norm

$$
\|\varphi\|_{H, T}=\inf _{\tilde{\varphi}}\|\tilde{\varphi}\|_{H},\|\psi\|_{H}=A_{H} \int|\hat{\psi}(\lambda)|^{2}|\lambda|^{d+2 H} d \lambda,
$$

Where $\tilde{\varphi}(t)$ is a finite function such that $\tilde{\varphi}(t)=\varphi(t), t \in \Delta_{T} ; \hat{\psi}(\lambda), \lambda \in R^{d}$ is the Fourier transform of $\psi(t)$. Obviously, we have $\varphi_{T}(0)=0, \varphi_{T}(1)=1$ for $t \in \Delta_{T} \backslash B_{1}$, and

$$
\begin{aligned}
\|\varphi\|_{H, T} & <\|f(|t| / T k)-f(|t|)\|_{H}<\|f(|t| / T k)\|_{H}+\|f\|_{H} \\
& =\left((T k)^{-H}+1\right)\|f\|_{H}<2\|f\|_{H} .
\end{aligned}
$$

\section{Acknowledgements}

I am very grateful to reviewer for careful reading of the paper and constructive comments.

\section{References}

F. Aurzada and S. Dereich. Universality of the asymptotics of the one-sided exit problem for integrated processes. Ann. Inst. Henri Poincaré Probab. Stat. 49 (1), 236-251 (2013). MR3060155.

F. Aurzada, N. Guillotin-Plantard and F. Pene. Persistence probabilities for stationary increment processes. ArXiv Mathematics e-prints (2016). arXiv: 1606.00236.

F. Aurzada and T. Simon. Persistence probabilities and exponents. In Lévy matters. $V$, volume 2149 of Lecture Notes in Math., pages 183-224. Springer, Cham (2015). MR3468226.

A. J. Bray, S. N. Majumdar and G. Schehr. Persistence and first-passage properties in nonequilibrium systems. Advances in Physics 62 (3), 225-361 (2013). DOI: 10.1080/00018732.2013.803819.

$\mathrm{X}$. Fernique. Regularité des trajectoires des fonctions aléatoires gaussiennes. In École d'Été de Probabilités de Saint-Flour, IV-1974, pages 1-96. Lecture Notes in Math., Vol. 480. Springer, Berlin (1975). MR0413238.

M. Lifshits. Lectures on Gaussian processes. Springer Briefs in Mathematics. Springer, Heidelberg (2012). ISBN 978-3-642-24938-9; 978-3-642-24939-6. MR3024389.

G. Molchan. Maximum of a fractional Brownian motion: probabilities of small values. Comm. Math. Phys. 205 (1), 97-111 (1999). MR1706900.

G. Molchan. Survival exponents for some Gaussian processes. Int. J. Stoch. Anal. pages Art. ID 137271, 20 (2012). MR2999109. 